\title{
Procesos generativos para morfologías bio-inspiradas en el tejido de madera del cactus
}

\section{Generative processes for bioinspired morphology in the wood tissue of cactus}

Ludovica Rossi

\begin{abstract}
Arquitecto, Doctor por la Escuela Técnica Superior de Arquitectura de Barcelona
\end{abstract} Universidad Politécnica de Cataluña

\section{RESUMEN}

ludovica.ro@gmail.com.

Se describe el procedimiento de simulación de las dinámicas de equilibrio de un sistema bioinspirado en la estructura de madera de los cactus. El diseño estudia la morfología funcional del referente organico por medio de la construcción de modelos. Las estrategias de simulación utilizan la búsqueda de patrones repetitivos para explorar procesos generativos y simular fenómenos complejos. Se describen los procedimientos de simulación realizados para definir las leyes de transformación que permiten generar la forma. Los modelos comparten el arquetipo de un rectángulo extruido según una directriz diferencial. El objeto geométrico resultante es la unidad base de la representación y caracteriza las propiedades topología del modelo. Las acciones sucesivas tratan: la iteración como proceso de repetición agregativo que permite al sistema crecer, y la aplicación de objetos deformadores como factores de variación de la foma que representa la capacidad de adaptación del tejido. Los resultados morfológicos varián según las acciones que generan la forma y los procesos asociados, mientras mantien las propiedades topologías. El diseño se entiende como un mapa genético donde los datos primitivos se van conectando unos con otros aumentando el nivel de jerarquía del sistema.

\section{ABSTRACT}

The simulation procedure of the equilibrium dynamics of a bio-inspired system in the wooden structure of cacti is described. The design studies the functional morphology of the organic referent through the construction of models. Simulation strategies use the search for repetitive patterns to explore generative processes and simulate complex phenomena. The simulation procedures performed to define the transformation laws that allow generating the shape are described. The models share the archetype of a rectangle extruded according to a differential guideline. The resulting geometric object is the base unit of the representation and characterizes the topology properties of the model. The successive actions deal with: iteration as an aggregative repetition process that allows the system to grow, and the application of deforming objects as factors of variation of the shape that represents the adaptability of the tissue. The morphological results vary according to the actions that generate the shape and the associated processes, while maintaining the topology properties. The design is understood as a genetic map where the primitive data are connected with each other, increasing the hierarchy level of the system.

PALABRAS CLAVES: arquetipo, biomímesis, Cinema 4D, deformación, topología.

KEY WORDS: archetype, biomimicry, Cinema 4D, deformation, topology.

FECHA DE RECEPCIÓN: 13/09/2020 | FECHA DE ACEPTACIÓN: 01/11/2020

DOI: http://dx.doi.org/10.30972/arq.0164549 


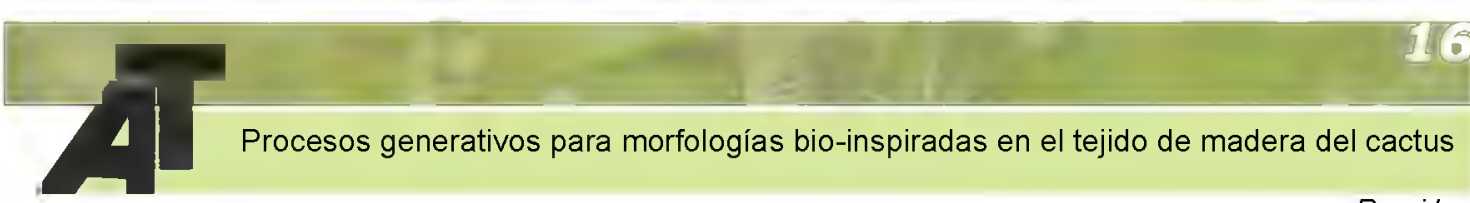

ARQUITECNO

RossiL

\section{INTRODUCCIÓN}

El presente artículo presenta los resultados del proceso de simulación digital de una morfologia bio-inspirada en la estructura de madera del cactus. El trabajo plantea como referentes orgánicos válidos, los cactus con tallo suculento, fotosintético y donde la disposición de las aréolas exteriores tiene una distribución romboidal. Bajo estas características se estudian ejemplares de la familia de las Cactáceas, de los géneros Echinopsis y Opuntia (Figura 1a y 1b).

Por su adaptación a la sequía, las cactáceas se caracterizan por la pérdida de resistencia mecánica a favor del tejido para el almacenamiento de agua y otros nutrientes. La estructura leñosa de la planta se encuentra en el interior del tallo y está formada por haces vasculares entrecruzados (Altesor \& Ezcurrawz, 2003). Estos están organizados en un conjunto de hélices de dos vías donde el esqueleto leñoso aparece como un retículo (Figura 1c).

La forma en la cual la planta se sostiene es un sistema complejo que basa su relación entre los tejidos de madera de los haces vasculares y los equilibrios de presión internos por turgencia, o sea intercambio de líquidos entre las células. Cuando la arquitectura hidráulica de la planta no es suficiente como soporte se desencadena un cambio de fase de la madera (Stevenson \& Mauseth, 2004) para garantizar el sostén de la planta. La planta pasa de una madera más elástica con mayor capacidad de acumulación de líquidos a una fibrosa más rígida y resistente. Esta respuesta morfogénica adaptativa está relacionada con el estrés del peso por el crecimiento de la planta y depende de las condiciones ambientales a las cuales ha sido expuesta.
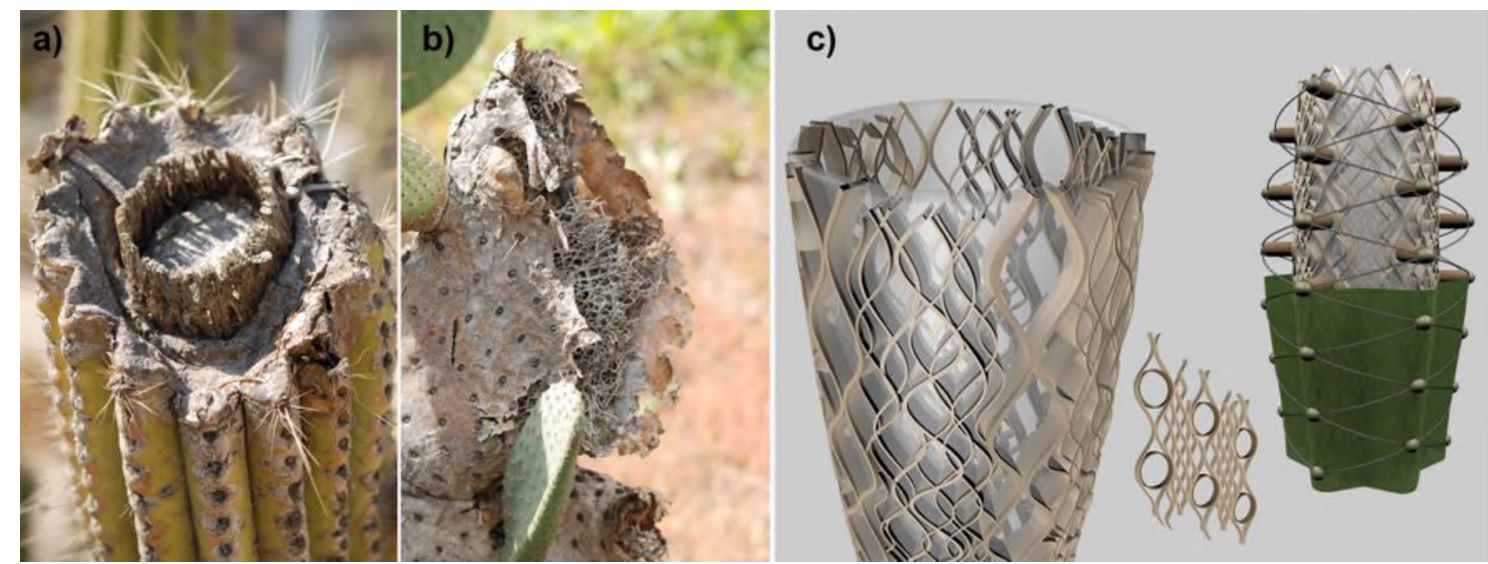

Figura 1: a) Cactus columnar del genero Echinopsis; b) Cactus del género Opuntia de crecimiento arbustivo

c) Simulación 3D de un cactus columnar, donde la estructura de sostén está interconectada con la distribución de las aréolas.

Fuente: Rossi, L. (2019). Diseño en flexión activa con neumáticos recuperados (tesis doctoral). ETSAB - UPC, Barcelona.

La morfología funcional del tejido de madera fibrosa de la planta viva trata los procesos biológicos de crecimiento y auto-organización. La adaptación mecánica se basa en variaciones de formas por flexibilidad de sus tejidos que se accionan mediante la implementación de inestabilidades elásticas por pandeo (Schleicher at all, 2014). Este mecanismo permite al cactus adaptarse a las condiciones medioambientales y responder a las necesidades de crecimiento del organismo.

La morfogenética del tejido vivo es el punto de partida para el diseño biomímetico. Las plantas, con sus innumerables principios de movimiento, son una fuente de inspiración prometedora. El principio de movimiento de estas estructuras biológicas se basa en principalmente en variaciones de forma por la flexibilidad de sus miembros (tejidos) y no en la unión de elementos rígidos con bisagras. Los sistemas de actuación de las plantas (sus movimientos) suelen estar optimizados evolutivamente para realizar un solo tipo de movimiento, a diferencia de la locomoción animal, que suele estar diseñada para una variedad de movimientos complejos. Además, las estructuras 


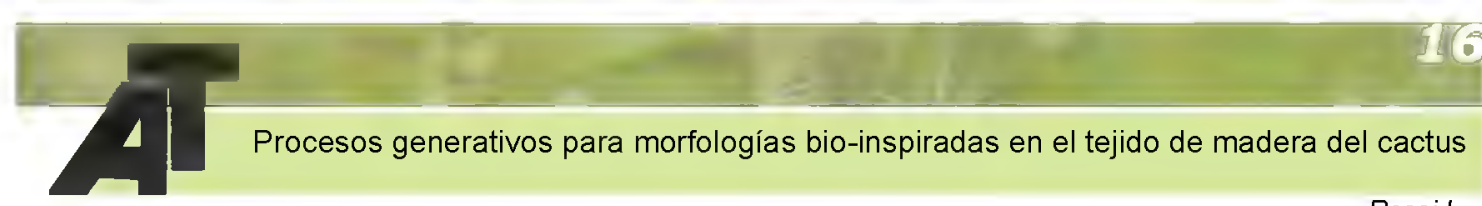

de las plantas se adaptan a menudo para condiciones de contorno similares, tales como las que afectan al diseño de las estructuras arquitectónicas (Lienhard at al., 2014).

\section{METODOLOGÍA}

La investigación sobre el referente natural aplica un enfoque biomimético Bottom-up, que sigue un proceso ascendente de abajo hacia arriba. Los conocimientos de las funciones y estructuras biológicas son punto de partida del diseño. Asimismo, la abstracción del mecanismo organico es un paso fundamental que permite separar las soluciones encontradas del referente biológico (Speck \& Speck, 2008). Este enfoque verifica cómo un modelo biológico puede ser un generador de conceptos y si estos pueden ser aplicados a la tecnología de forma creativa.

La metodología de trabajo reproduce los mecanismos biológicos del tejido vivo de la planta por medio de la construcción de los modelos físicos en campo elástico (y no rígido). Se analiza la biomecánica y la morfología funcional del tejido vivo de la madera del cactus, entendido cómo el sistema biológico de referencia (Figura 2a). Cada fenómeno natural por cuanto simple es en realidad compuesto, y cada efecto visible es la suma de un número infinito de acciones subordinadas, donde el crecimiento y la forma son, bajo todos los aspectos, de naturaleza compuesta (Thompson, 1969, 292). Para separar los fenómenos involucrados, las construcciones de modelos físicos permiten observar la relación dinámica entre las fibras y el comportamiento del tejido en su conjunto. Los modelos físicos (Figura 2b) ponen a prueba la funcionalidad del mecanismo separando progresivamente todos los elementos que parecen no estar relacionados con el mecanismo. Siguiendo este enfoque, se pueden reducir las partes constituyentes que juegan un papel clave en el mecanismo. Esto es de particular importancia porque el conocimiento sobre los componentes básicos que se necesitan para un mecanismo abre la puerta para su uso creativo (Lienhard at al., 2014). Esta visión amplía la libertad de diseño y permite conceptos más allá de la mímica directa y así poner en práctica de forma abstracta los principios biológicos de adaptabilidad y crecimiento.

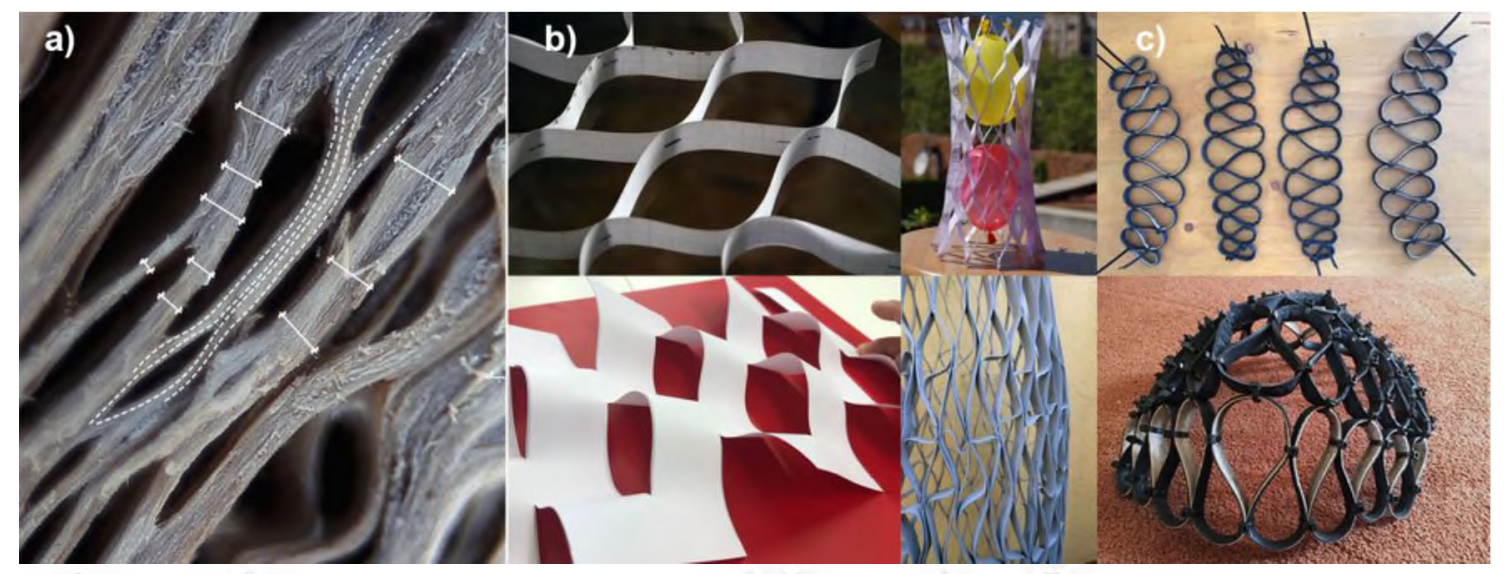

Figura 2: a) Tejido de madera seco de cactus con la variación del recorrido curvilineo y del ancho de las fibras; b) Modelos a pequeña escala realizados en papel; c) Modelos en banada de rodadura de neumáticos de bicicleta recuperados. Fuente: Rossi, L. (2019). Diseño en flexión activa con neumáticos recuperados (tesis doctoral). ETSAB UPC, Barcelona.

La reproducción física del tejido permite verificar los mecanismos morfogenéticos y poner en relaciōn la observación de la plantilla biológica estática (tejido seco) con el comportamiento dinámico del tejido vivo. La implementación técnica del mecanismo biológico en forma de modelos fisicos no se encuentra al final, sino más bien en medio de un proceso de diseño iterativo en el que la retroalimentación de la construcción puede llegar tan lejos, como para fomentar la comprensiôn del modelo biológico (Lienhard at al., 2014). Por diferentes niveles de aproximación, 


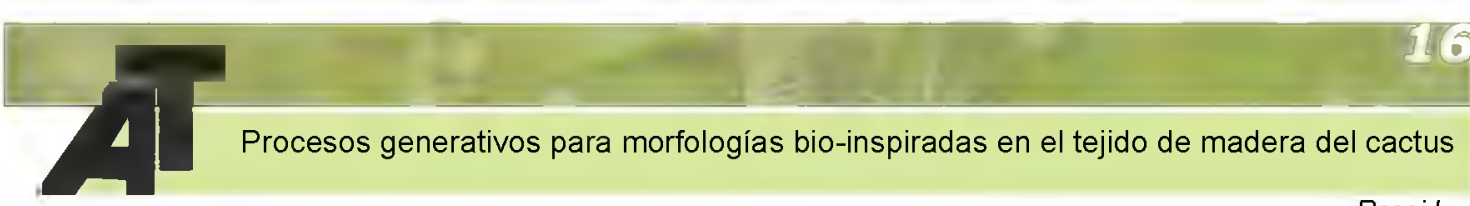

ARQUITECNO

Rossi L

los modelos permiten estudiar las relaciones entre la morfología del tejido estructural de madera y la fisiología de la planta. En este intercambio de información entre estudio de las soluciones biológicas y la construcción de los modelos para la comprobación del mecanismo bio-inspirado, surgen nuevos hallazgos que también contribuyen a una mejor comprensión del funcionamiento de los generadores de conceptos biológicos (el referente orgánico). Se activa una dinámica de "biomimética inversa", y esto requiere una nueva investigación de las muestras biológicas para lograr una mejora continua en la comprensión del modelo técnico (Speck at al., 2017).

En este proceso de diseño iterativo los modelos físicos permiten encontrar un sistema con un adecuado equilibrio entre rigidez y elasticidad. El procedimiento permite la abstracción del tejido resistente de la planta y la conceptualización del referente orgánico. Se define la estructura del cactus como una sumatoria de elementos lineares que se desarrollan en la misma dirección del crecimiento de la planta y se expanden por agregación de tejidos. Esta interpretación define la forma de la franja o fibra como el elemento morfológico de base (Figura 2c). Se determina el arquetipo que permite abandonar el refernte organico y sistematizar la información para su aplicación técnica.
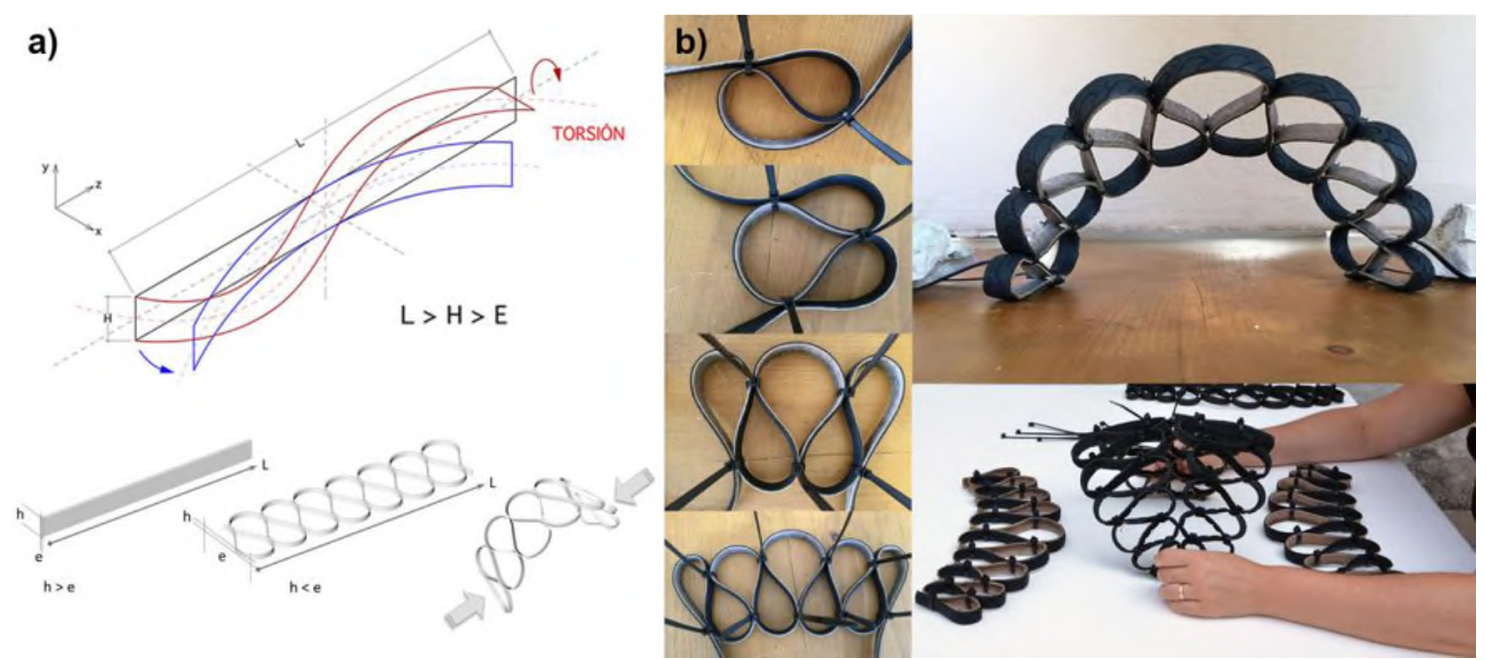

Figura 3: a) Características geometricas y variación morfológica de la fibra donde se modifica la relación geométrica entre la altura y el ancho del elemento; b) Construcción de modelos en fibras elásticas pre-deformadas en petálos alternados.

Fuente: Rossi, L. (2019). Diseño en flexión activa con neumáticos recuperados (tesis doctoral). ETSAB - UPC, Barcelona.

\section{DESARROLLO}

\section{Simulación digital}

El estudio morfológico es definido en relación a la capacidad de adaptación que tiene el tejido orgánico vivo. La interpretación realizada sobre la fibra del cactus ha priorizado una visión lineal de la misma. El tejido se evalua como una suma de células, que se agregan progresivamente en la dirección del crecimiento de la planta. El elemento morfológico de base se define como una fibra que tiene en una dirección principal respecto a las otras dos: $\mathrm{L}>\mathrm{H}>\mathrm{E}$ (Figura 3a). Asimismo, la morfología general se desarrollada a partir de las dinámicas de equilibrio obtenidas en los modelos físicos conformados por franjas pre-deformadas en pétalos alternados (Figura 3b). Este entorno de trabajo se caracteriza por la relación que existe entre objeto y materia, y donde la deformación es parte relevante en la definición del ámbito geométrico.

El procedimiento de simulación definine las leyes de transformación que permiten generar la forma y el entorno digital permite codificar la geometría de modelos elásticos construidos. Se 


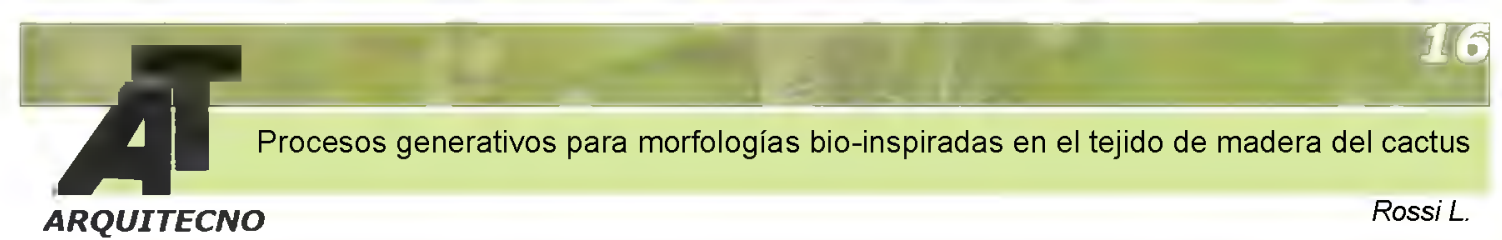

ARQUITECNO

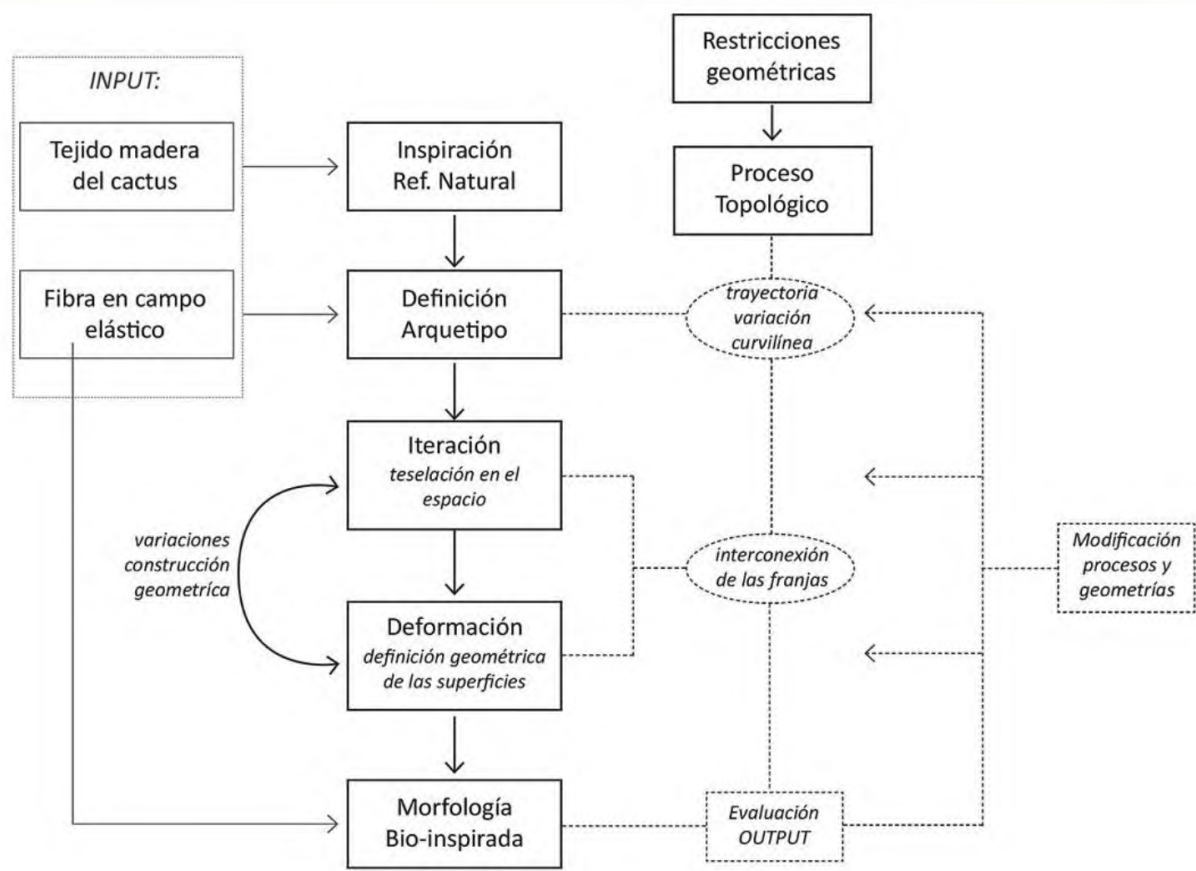

Figura 4: Esquema del proceso del diseño generativo en relación a los factores iniciales y las restricciones geométricas. Fuente: Rossi, L. (2019). Diseño en flexión activa con neumáticos recuperados (tesis doctoral). ETSAB - UPC, Barcelona

La problemática es representar el producto final en términos de los procesos que lo generan, como una secuencia de operaciones bien definidas, y donde el modelo virtual debe estar dotado de puntos de mutación en los lugares correctos (De Landa, 2002). Esto implica implementar un análisis topológico para la génesis de la forma. Los espacios topológicos son espacios abstractos en los que las nociones habituales de longitud, área y volumen no tienen sentido alguno (De Landa, 2009, 10). La forma es el resultado de una conexión de datos abstractos en un sistema primario y los diseños realizados se estructuran de manera que siempre respetarán ciertas invariantes topológicas (Figura 4), como la trayectoria curvilínea de las fibras o la conectividad entre las franjas en los modelos.

La simulación virtual establece las leyes de transformación que se aplican. Las propiedades geométricas de las fibras definen el arquetipo inicial, que incide en el resultado morfológico global. Estudiando la forma se establecen las relaciones entre las variaciones aplicadas (extrusión, iteración, deformación). El diseño es como un mapa genético donde se parte de datos o valores primitivos que se conectan unos con otros aumentando de nivel en su jerarquía estructural. Es decir, la información primaria irá evolucionando conforme se conecta con otros conjuntos de información y de ese proceso nacerá un nuevo conjunto de datos que llevará la información heredada de los pasos anteriores. La simulación virtual se compone de una serie de procesos que generarán el último componente del sistema como resultado final del diseño (Frogheri at al., 2017).

Las operaciones geométricas implementadas para generar la forma orgánica se dividen en tres grupos principales: el arquetipo como representación del elemento de base, las operaciones de iteración y la aplicación de las deformaciones como procedimiento de variación de la forma. La introducción de los factores de trasformación (en diferentes fases del diseño según caso) permite definir la deformación como la capacidad de adaptación del tejido orgánico.

Entre los diferentes modelos virtuales desarrollados no hay una variación de los parámetros que permiten su construcción, por otra parte, el el procedimiento paramétrico aplicado genera variaciones en los resultados, variando el orden en el cual se explicitan los parámetros. En la 


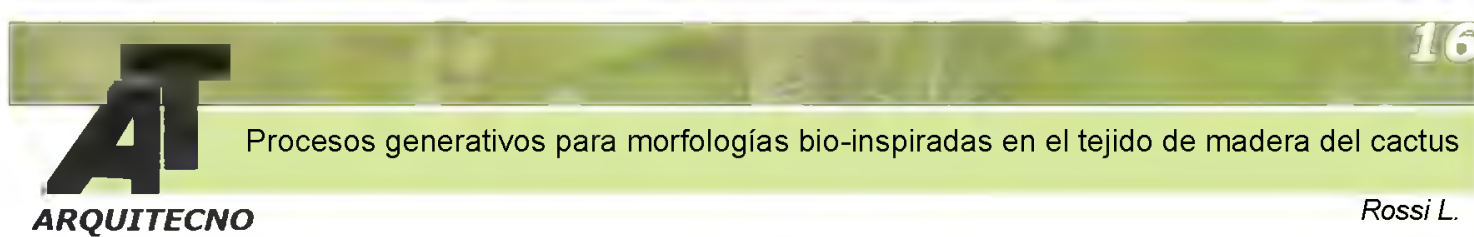

descripción de los casos de estudio se observa cómo la variación en el orden de unas operaciones geométricas lleva a obtener diferentes resultados morfológicos en el espacio digital.

\section{Construcció de los modelos (arquetipo - deformación - iteración)}

Todos los modelos se basan en el diseño algorítmico y comparten la acción de iterar el elemento de base (la franja) en el espacio, para llegar al sistema general. La franja deviene entonces al arquetipo de los modelos virtuales. Este es el patrón inicial del cual deriva la generación de formas complejas en el desarrollo del diseño paramétrico.

La representación del modelo virtual empieza por definir cómo representar la franja (arquetipo). Esta se codifica por la transformación de la figura base de un rectángulo, que es extruida (SweepNURBS) según una trayectoria curvilínea, es decir según un crecimiento en dos direcciones. La franja pre-deformada según la directriz de los pétalos alternados representa un primer orden de la variación de la forma que es implícito en el arquetipo y que define la topología del modelo.

En las simulaciones realizadas se distinguen el proceso de deformación como construcción geométrica del diseño implícita en la representación del arquetipo, y la deformación aplicada como cambio morfológico. En este segundo caso la deformación es un parámetro abstracto que se aplica al diseño para obtener el conjunto de las variaciones de la forma necesarias para reproducir un efecto similar al observado en el comportamiento físico del material. Estas deformaciones son variaciones sobre el objeto representado inicialmente, la forma inicial, que topológicamente es la misma, adquiere diferentes valores con la aplicación de uno o más deformadores. Estos doblan el objeto y generan la variación de la forma inicial.

En el espacio de diseño virtual, la deformación se concibe como una redistribución proporcional de puntos a lo largo de una dirección. Las nociones de "redistribución" y "dirección" implican la existencia del tiempo como una medida de comparación. Para concebir el cambio dictado por la deformación, se reconoce el estado previo de la forma, antes de que se produzca la deformación. La deformación se entiende, entonces, como un cambio relativo de un estado inicial, y como punto de referencia se necesita un arquetipo (de forma análoga pero diferente a la clonación) para evaluar el grado de deformación (Terzidis, 2003).

Definida la unidad base de la fibra se aplica un proceso de repetición agregativo que permite al sistema crecer. La iteración (Cloner) de la fibra en el espacio es una trasformación con diferentes niveles de complejidad y, dependiendo del modelo, se basa en las operaciones de traslación, simetría o rotación. La operación de iteración es un crecimiento diferencial del sistema en el espacio (Patiño Mazo at al., 2017). Las copias están todas vinculadas al objeto inicial dibujado y a sus posibles variaciones. Modificando el objeto originario cambian todos los demás. La clonación se basa en la interpretación y, como tal, debe estar asociada a un arquetipo (la franja). Es un método implícito de alteración que se basa en el comportamiento en lugar de la forma. (Terzidis, 2003).

En los modelos realizados la aplicación de la variación de la forma (deformaciones) puede ser previa o posterior a la iteración del arquetipo de la franja, según el procedimiento se obtienen diferentes resultados como se detalla más adelante.

\section{DISCUSIÓN DE RESULTADOS}

Por las características constructivas del prototipo formado en franjas dobladas en petalos alternados (Figura 2 c y $3 b$ ) el interés de la simulación virtual está en la subdivisión de las acciones que permiten definir la configuración general del sistema. La franja se dibuja a partir de la extrusión de la sección rectangular según la trayectoria en pétalos de dimensiones variables. La 


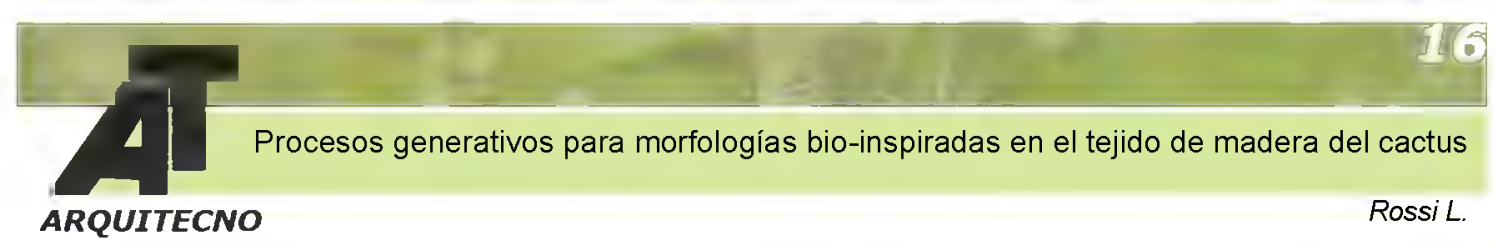

primera deformación aplicada a la franja en los modelos físicos queda implícita en la representación del objeto base que define el patrón partida de este modelo virtual (Figura 5a).

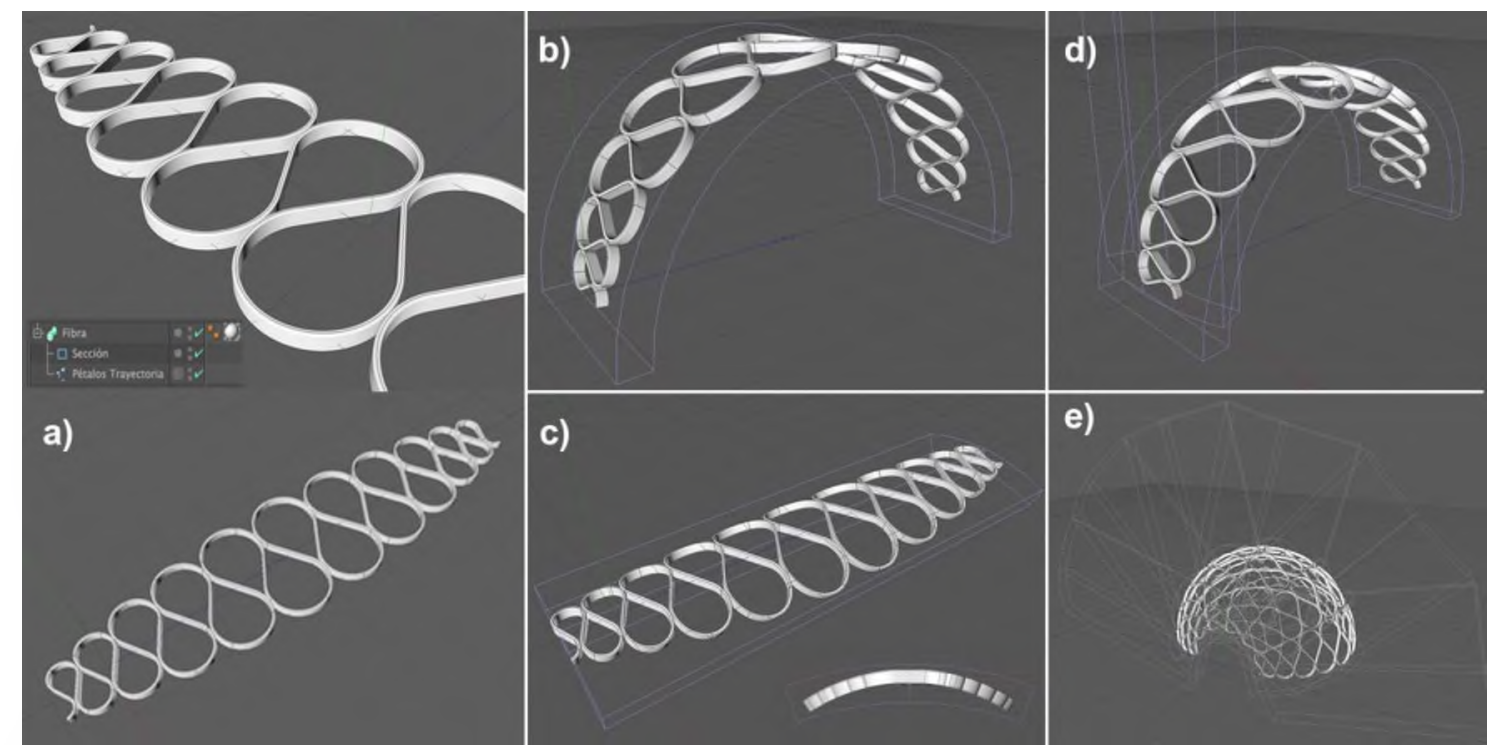

Figura 5: Proceso generativo cúpula franja en petálos alternados: a) Fibra por extrución sección rectangular; b) Arco por pliegue $180^{\circ}$; c) Dos pliegues simétricos de $20^{\circ}$ para el efecto inflado; d) Aplicación conjunta de los dos niveles de deformación; e) Operación de clonación por rotación de la fibra deformada para la construcción de la cúpula.

Fuente: Rossi, L. (2019). Diseño en flexión activa con neumáticos recuperados (tesis doctoral). ETSAB - UPC, Barcelona.

El arquetipo del espacio virtual está representado como la franja pre-deformada del objeto material. El elemento así dibujado es plano y para generar la forma en arco se implementa una variación de forma que permite un pliegue de $180^{\circ}$ (Figura 5b). El diseño aplica el objeto deformador doblar (Ben Object) para curvar la franja en forma de arco. Además, es necesario agregar otro nivel de deformación (Figura 5c), para obtener la doble curvatura generada en la morfología de la cúpula. En el modelo físico a la vez que se unen las franjas pre-deformadas se van generando las deformaciones que permiten al sistema asumir la forma esférica (Figura 3b). En le modelo virtual, una vez dibujada la franja, se aplican dos deformaciones según dos directrices perpendiculares entre sí, para luego clonar el elemento en el espacio y obtener la configuración final de una semiesfera (Figura $5 \mathrm{~d}$ ).

La doble curvatura de la cúpula se obtiene aplicando las dos deformaciones conjuntamente (Figura 5d). La primera (Figura 5b) proporciona la forma en arco, aplicando la variación de la forma respecto al eje de desarrollo longitudinal (z). El segundo nivel de curvatura (Figura $5 \mathrm{c}$ ) se implementa aplicando el mismo tipo de deformador "pliegue" según el eje trasversal $(x)$ de forma simétrica. Esta deformación permite obtener el efecto "inflado" del sistema elástico.

Susesivamente por medio de una operación de clonación del elemento deformado, se reproduce el acoplamiento del elemento en arco que forman la cúpula del modelo físico. La iteración por rotación del elemento deformado según las dos curvaturas (ejes longitudinal y transversal) completa las operaciones geométricas que definen la morfología de la cúpula (Figura 5e). La operación de reiteración (Cloner) indica el número de fibras y sus posiciones en el espacio de representación. 


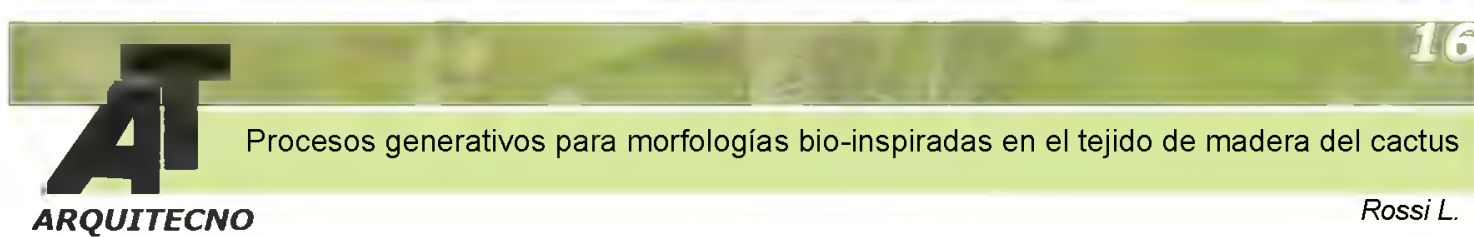

respecto a la dimensión de los procesos a ellos asociados. Esto implica evaluar la técnica utilizada desde una dimensión progresiva, que explicita la diferencia entre lo que se hace y lo que ocurre. Donde el primero hace referencia a la ejecución y el segundo a la secuencia de acontecimientos que determinan o permiten llegar a una cierta configuración espacial.

\section{BIBLIOGRAFIA}

Altesor, A., Ezcurrawz, E. (2003). Functional morphology and evolution of stem succulence in cacti. en Journal of Arid Environments, Vol. 53 Núm. 4, Ed. ELSEVIER, 557-567.

Castro Henriques, G., Nóbrega, G., Passaro, A. (2017). Tentáculos: Recriando criaturas híbridas, analógicodigitais, en Actas SIGraDi 2017, XXI Congreso de la Sociedad Ibero-americana de Gráfica Digital, 22-24 noviembre, 2017, Concepción, Chile.

De Landa, M. (2002). Deleuze and the use of the genetic algorithm in architecture. en Architectural Design, Vol. 72 Núm. 1, Ed. John Wiley \& Sons Ltd, 9-12.

De Landa, M. (2009). Emergencia, causalidad y realismo. en Artnodes, Núm. 9, Ed. Universitat Oberta de Catalunya, 4-14.

Frogheri, D., Estévez, A. T., Meneses-Carlos, F. (2017). Morfologías resilientes: Desde lo digital a la materia. en Actas SIGraDi 2017, XXI Congreso de la Sociedad Ibero-americana de Gráfica Digital, 22-24 noviembre, 2017, Concepción, Chile.

Hossdorf, H., Hernández, C. B. (1972). Modelos reducidos. Madrid: Ed. IETcc.

Lienhard, J., Schleicher, S., Knippers, J. (2014). Bio-inspired, flexible structures and materials. En Biotechnologies and Biomimetics for Civil Engineering Edition (cap.11). Suisa: Springer Ed.

Patiño Mazo, E., Valencia-Escobar, A., Torreblanca Díaz, D. A., Zuleta Gil, A. (2017). Proceso Generativo de Texturas Paramétricas Bioinspiradas: Modelo Metodológico de Experimentación. en Actas SIGraDi 2017, XXI Congreso de la Sociedad Ibero-americana de Gráfica Digital, 22-24 noviembre, 2017, Concepción, Chile.

Rossi, L. (2019). Diseño en flexión activa con neumáticos recuperados (tesis doctoral). ETSAB UPC, Barcelona. Recuperado de https://www.tdx.cat/handle/10803/668046\#page=1

Schleicher, S., Lienhard, J., Poppinga, S., Speck, T., Knippers, K. (2014). A methodology for transferring principles of plant movements to elastic systems in architecture. en ComputerAided Design, Vol. 60, Ed. ELSEVIER, 105-117.

Speck, O., Speck, D., Horn, R., Gantner, J., Sedlbauer, K. P. (2017). Biomimetic bio-inspired biomorph sustainable? An attempt to classify and clarify biology-derived technical developments. en Bioinspiration \& Biomimetics, Vol 12 Núm. 1, Ed. IOP Publishing Ltd, 115.

Speck, T., Speck, O. (2008). Process sequences in biomimetic research. Design and Nature, IV Comparing Design in Nature with Science and Engineering, 3-11.

Stevenson, J. F., Mauseth J. D. (2004). Effects of Environment on Vessel Characters in Cactus Wood. en International Journal of Plant Sciences, Vol. 165, Núm. 3, Ed. The University of Chicago Press, 347-357.

Terzidis, K. (2003). Expressive Form. A conceptual approach to computational design. London y New York: Ed. Spon Press Taylor \& Francis Group, London y New York.

Terzidis, K. (2017). The Intricacy of the Otherness. en Archi DOCT - Algorithmic Thinking, Vol. 4 Núm. 2, Ed. Enhsa, 11-27.

Thompson D'Arcy, W. (1969). Crescita e Forma. Torino: Ed. Universale Scientifica Boringhieri. 